\title{
ESTUDOS DE QSAR-3D PARA UMA SÉRIE DE ANÁLOGOS À TIMIDINA COM ATIVIDADE TUBERCULOSTÁTICA
}

Renata Vieira BUENO ${ }^{11 a^{*}}$

Rodolpho Campos BRAGA ${ }^{\text {1a }}$

Ney Ramos TOLEDO ${ }^{2 a}$

Carolina Horta ANDRADE ${ }^{3 a}$

RESUMO: A tuberculose (TB) é uma doença infecto-contagiosa crônica com altas taxas epidemiológicas. O surgimento de cepas multi- e extensivamente resistentes aos fármacos utilizados no tratamento, bem como a longa duração e os efeitos colaterais, tornam urgente o desenvolvimento de novos tuberculostáticos. Além disso, a busca por novos alvos de ação se faz necessária, já que os antimicobacterianos utilizados na terapia anti-TB têm como alvo apenas um pequeno número de enzimas relacionadas a funções essenciais do microrganismo. Enzimas que atuam na síntese de DNA, como a timidina monofosfato quinase (TMPK), são alvos promissores para o planejamento racional de novos tuberculostáticos. Neste trabalho, estudos de QSAR-3D foram realizados através da aplicação da Análise Comparativa dos Campos Moleculares (CoMFA), com o objetivo de elucidar os requisitos estruturais relevantes à atividade biológica e gerar modelos de QSAR-3D para previsão da atividade de compostos não sintetizados, considerando dados quantitativos de atividade biológica e a interação com a enzima TMPKmt de 106 análogos de timidina obtidos a partir da literatura. Modelos robustos e com boa capacidade de predição foram obtidos, confirmando a importância de grupos com maior densidade eletrônica e estericamente desimpedidos no anel 2. Os resultados obtidos com os análogos da timidina, por meio de processos racionais de planejamento forneceram dados importantes para a busca de candidatos a novos fármacos tuberculostáticos.

Palavras-chave: Tuberculose. TMPK. Análogos de timidina. Tuberculostáticos potenciais. QSAR-3D.

\begin{abstract}
Tuberculosis (TB) is a chronic infectious disease with high epidemiological rates. The emergence of multidrug and extensively drug-resistant TB strains, as well as the long-term treatment and the side effects, become urgent the need for the development of new anti-tuberculosis drugs. Furthermore, the search for new targets is also necessary; as the current antimycobacterial drugs have just a small number of enzymes related to essential functions of the mycobacteria as targets. TMPKmt is an attractive target for the design of new antituberculosis agents since this enzyme is essential for DNA replication. In this work, we used the 3D-QSAR, by applying the Comparative Molecular Field Analysis (CoMFA), in order to elucidate the structural requirements relevant to the biological activity and to generate 3D-QSAR models for predicting the biological activity of compounds not yet synthesized, considering quantitative biological data and the interaction with TMPKmt of 106 thymidine analogues obtained from the literature. Robust and significant statistical models were obtained, confirming the importance of groups with higher electronic

\footnotetext{
${ }^{1}$ Alunos de mestrado acadêmico do Programa de Pós-Graduação em Ciências Farmacêuticas da Universidade Federal de Goiás - UFG, Goiânia, Goiás

${ }^{2}$ Aluno de graduação em Farmácia na Faculdade de Farmácia da Universidade Federal de Goiás - UFG, Goiânia, Goiás

${ }^{3}$ Professor Doutor, Adjunto I, da Faculdade de Farmácia da Universidade Federal de Goiás - UFG

${ }^{a}$ Laboratório de Planejamento de Fármacos e Estudos do Metabolismo por Modelagem Molecular (LabMol), Faculdade de Farmácia, Universidade Federal de Goiás, 74605-222 Goiânia - GO, Brasil

* e-mail: buenorvieira@gmail.com
} 
density and less bulky near ring 2 . The studies herein developed with thymidine analogues through rational drug design lead to important data towards the search of new candidates as new anti-tuberculosis drugs.

Keywords: Tuberculosis. TMPK. Thymidine analogues. Potential tuberculostatic agents. 3D-QSAR.

\section{INTRODUÇÃO}

A tuberculose (TB) é uma doença infecto-contagiosa crônica com prevalência mundial de 12.000.000 de casos, segundo estimativa de 2011 (WHO, 2011). O surgimento de cepas multi- e extensivamente resistentes aos fármacos utilizados no tratamento, a longa duração e os efeitos colaterais tornam urgente o desenvolvimento de novos tuberculostáticos (SOUZA; VASCONCELOS, 2005).

O planejamento racional de novos fármacos pode ser realizado por diversas estratégias da Química Farmacêutica Medicinal, dentre elas a utilização de ferramentas computacionais que relacionem dados biológicos à estrutura química. Neste contexto, estudos de QSAR (do inglês Quantitative Structure-activity

Relationshisps) constituem ferramentas úteis à descoberta de novos candidatos a protótipos de fármacos.

Enzimas envolvidas na síntese de DNA, como a timidina monofosfato quinase (TMPK), são alvos promissores no planejamento racional de novos tuberculostáticos. A TMPK catalisa a fosforilação reversível de desoxitimidina monofosfato (dTMP) à desoxitimidina difosfato (dTDP). É, portanto, uma enzima essencial para a síntese de DNA e replicação celular (FAMILIAR et al., 2008). A identidade entre a TMPK do Mycobacterium tuberculosis (TMPKmt) e a TMPK humana (TMPKh) é de apenas $22 \%$, o que atrelado à identificação do inibidor competitivo da TMPKmt, $\quad$ 3'-azido-2'-desoxitimidina monofosfato torna esse alvo promissor para o planejamento racional de novos tuberculostáticos (MUNIER-LEHMANN et al., 2001).

Diante da necessidade de se desenvolver novas opções terapêuticas, o presente trabalho objetivou elucidar os requisitos estruturais relevantes à atividade biológica e gerar modelos de QSAR-3D que possibilitem prever a atividade de compostos não sintetizados, considerando dados quantitativos de atividade biológica e a interação com a enzima TMPKmt de 106 análogos de timidina selecionados da literatura (FAMILIAR et al., 2008; FAMILIAR et al., 2010; POCHET et al., 2003; VAN DAELE et al., 2006; VAN DAELE et al., 2007; VANHEUSDEN et al., 2002, 2003a, b)

\section{METODOLOGIA}

Os 106 análogos de timidina foram obtidos da literatura (FAMILIAR et al., 2008; FAMILIAR et al., 2010; POCHET et al., 2003; VAN DAELE et al., 2006; VAN DAELE et al., 2007; VANHEUSDEN et al., 2002, 2003a, b). Cabe ressaltar que tais compostos foram submetidos ao mesmo protocolo farmacológico para obtenção dos 
valores de atividade biológica, expressa em $K$ i. Os valores de $K_{\mathrm{i}}(\mu \mathrm{M})$ foram convertidos em $\mathrm{p} K_{\mathrm{i}}\left(-\log K_{\mathrm{i}}\right)$, com valores que variaram de 2,721 a 6,569 .

Os estudos de QSAR-3D foram realizados em sistema operacional Linux Ubuntu v. 11.10. Foram utilizados os softwares: SYBYL-X 1.2 (Tripos, Inc.); OMEGA, FRED, ROCS, VIDA e QUACPAC (OpenEye Scientific Software, Inc.). Os compostos foram divididos em dois conjuntos, o de treinamento e o de avaliação.

O número de moléculas do conjunto de avaliação em relação ao total foi de $21,7 \%$. A validação interna e externa dos modelos gerados foi feita através do método de regressão por quadrados mínimos parciais (PLS, do inglês Partial Least Squares). Através do método de validação cruzada leave-one-out (LOO) foi realizada a validação interna. Para validação externa foram empregadas as moléculas do conjunto de avaliação.

Utilizou-se como método de QSAR3D o CoMFA do pacote SYBYL-X 1.2 (Tripos Inc., St. Louis, USA). As estruturas 3D dos compostos e seus confôrmeros foram gerados no programa OMEGA. As cargas foram calculadas pelo método semi-empírico AM1-BCC, através do programa QUACPAC. O alinhamento estrutural, necessário para geração de modelos do CoMFA, foi realizado por duas abordagens: alinhamento pela estrutura comum (SYBYL-X) e alinhamento baseado nas coordenadas do ancoramento (docking) do ligante mais ativo no sítio ativo da enzima TMPKmt, utilizando o programa FRED. As moléculas alinhadas foram inseridas na caixa $3 \mathrm{D}$ virtual $\mathrm{e}$ foram calculados os campos de interação estéricos e eletrostáticos. Foram empregados o campo de força Tripos e um átomo de prova, o carbono com hibridação $\mathrm{sp}^{3}$ e carga +1 . A correlação estatística entre os valores das energias de interação em cada ponto da caixa e a atividade biológica, por sua vez, foi feita pela regressão por PLS.

\section{RESULTADOS E DISCUSSÃO}

Após o alinhamento das 83 moléculas do conjunto de treinamento, geração e otimização dos modelos de CoMFA, foram obtidos os resultados estatísticos apresentados na Tabela 1.

Ambos modelos gerados apresentam boa consistência interna, com valores de $q^{2}$ superiores a 0,5. O modelo de CoMFA gerado pelo alinhamento da estrutura comum (1) apresentou melhores resultados estatísticos do que o modelo obtido a partir do alinhamento baseado no docking do ligante mais ativo no sítio ativo da enzima TMPKmt (2).

A Figura 1 apresenta os valores experimentais e previstos de $\mathrm{p} K i$ para os dois melhores modelos de QSAR-3D gerados, para os conjuntos de treinamento e avaliação, confirmando a consistência interna e externa destes modelos.

O alinhamento dos compostos é etapa essencial para a geração de modelos de 
CoMFA $\quad\left(\right.$ COMFA $^{\mathrm{TM}}$ MANUAL, 2006).

Assim, a estratégia de alinhamento utilizada influencia na qualidade do modelo obtido. Neste trabalho, o melhor modelo de QSAR3D foi obtido através do alinhamento baseado na estrutura comum (1).

Apesar dos dois modelos não apresentarem percentuais de contribuição estérica e eletrostática semelhantes, as informações obtidas na análise dos mapas de contorno do CoMFA são coerentes. Os mapas de contorno estérico de ambos modelos mostraram que a presença de grupos volumosos nas posições a e b e na região do anel 2 (Figura 2) é favorável à atividade biológica, porém grupos volumosos próximos ao grupo sulfona são desfavoráveis (Figura 2).

Os mapas de contorno de campos

Tabela 1 - Resultados estatísticos dos melhores modelos do CoMFA

\begin{tabular}{cccccccc}
\hline Alinhamento & $q^{2}$ & $N$ & $r^{2}$ & $S E E$ & \multicolumn{2}{c}{ Contribuição } & Capacidade \\
& & & & & Estérica & Eletrostática & preditiva \\
\hline Modelo (1) & 0,70 & 6 & 0,83 & 0,39 & 0,79 & 0,21 & $73,9 \%$ \\
& & & & & & & \\
Modelo (2) & 0,67 & 3 & 0,79 & 0,42 & 0,39 & 0,61 & $69,6 \%$
\end{tabular}

$q^{2}$ : coeficiente de correlação de validação cruzada (LOO); $N$ : número ótimo de componentes PLS; $r^{2}$ : coeficiente de correlação linear; SEE: erro padrão estimado;

Fonte: Autor

Figura 1 - Valores experimentais e previstos de pKi dos conjuntos de treinamento (quadrados) e de avaliação (triângulos) para os modelos de CoMFA gerados pelos alinhamentos 1 (A) e 2 (B), respectivamente.

A
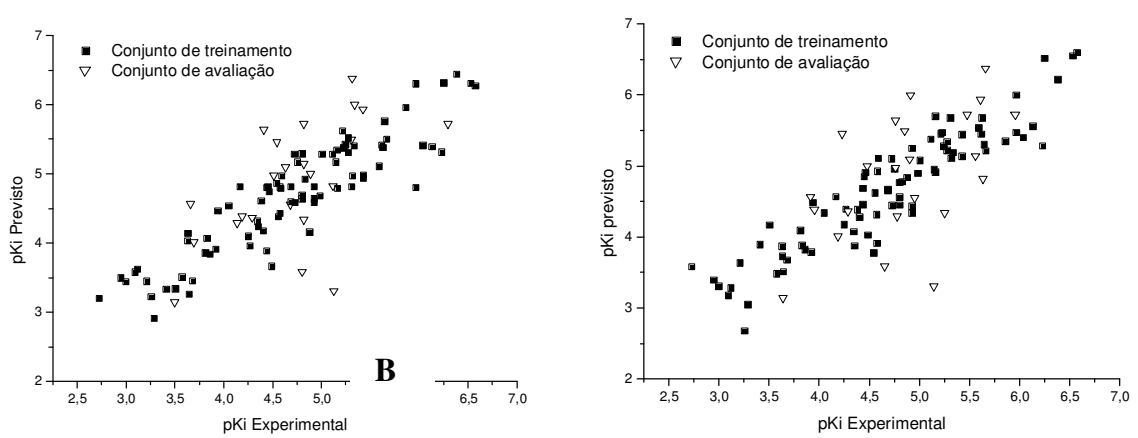

Fonte: Autor 
Figura 2 - Representação 2D da estrutura química do composto mais ativo (A), Mapas de contorno 3D CoMFA de campos estéricos e eletrostáticos para o modelo 1 (B) e para o modelo 2 (C).

A

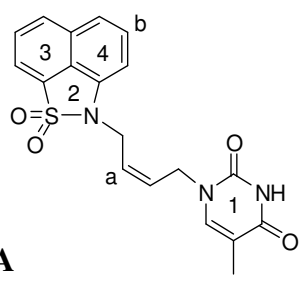

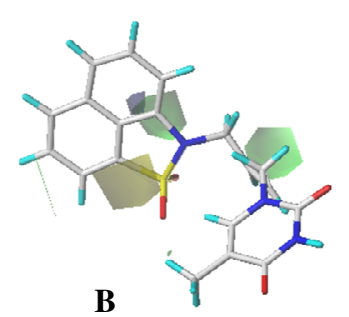

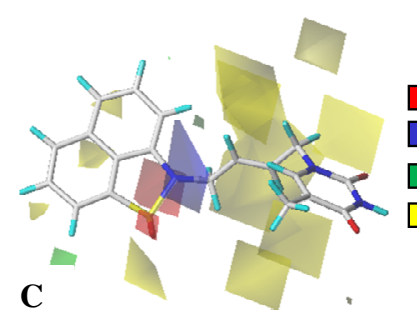

Densidade eletrônica favorável à atividade biológica Densidade eletrônica desfavorável à atividade biológica Efeito estérico favorável à atividade biológica

Efeito estérico desfavorável à atividade biológica

Fonte: Autor

\section{CONCLUSÃO}

Modelos de QSAR-3D gerados através dos estudos de CoMFA apresentaram consistência interna e externa quanto à capacidade preditiva, confirmando a importância de grupos com maior densidade eletrônica e estericamente desimpedidos no anel 2.

Os modelos de QSAR-3D O modelo obtido pelo alinhamento baseado na estrutura comum (1) apresentou melhores resultados estatísticos e capacidade preditiva que o modelo gerado pelo alinhamento baseado nas coordenadas de ancoramento do ligante mais ativo (2), o que demonstra a influência da estratégia de alinhamento na obtenção de modelos consistentes.

Os modelos gerados neste estudo podem ser úteis para a previsão da atividade biológica de compostos ainda não testados, e podem ser utilizados como uma ferramenta para o planejamento racional de candidatos a novos fármacos tuberculostáticos.

\section{AGRADECIMENTOS}

Os autores agradecem ao Conselho Nacional de Desenvolvimento Científico e Tecnológico (CNPq) e Coordenação de Aperfeiçoamento de Pessoal de Nível Superior (CAPES) pelo financiamento da pesquisa.

\section{REFERÊNCIAS BIBLIOGRÁFICAS} CoMFA $^{\mathrm{TM}}$ MANUAL, SYBYL 7.2, Tripos Inc., St. Louis, MO, 2006.

FAMILIAR, O.; MUNIER-LEHMANN, H.; NEGRI, A.; GAGO, F.; DOUGUET, D.; RIGOUTS, L.; HERNÁNDEZ, A-I; CAMARASA, M-J.; PÉREZ-PÉREZ, M-I. Exploring acyclic nucleoside analogues as inhibitors of Mycobacterium tuberculosis thymidylate kinase, ChemMedChem, v. 3, p. 1083-1093, 2008.

FAMILIAR, O.; MUNIER-LEHMANN, H.; AÍNSA, J. A.; CAMARASA, M-J.; PÉREZPÉREZ, M-I. Design, synthesis and inhibitory activity against Mycobacterium tuberculosis thymidine monophosphate kinase of acyclic nucleoside analogues with a distal imidazoquinolinone, European Journal of Medicinal Chemistry, v. 45, p. 5910-5918, 2010. MUNIER-LEHMANN, H.; CHAFOTTE, A.; POCHET, S.; LABESSE, G. Thymidilate kinase of Mycobacterium tuberculosis: a 
chimera sharing properties common to eukaryotic and bacterial enzymes. Protein Science, v. 10, p. 1195-1205, 2001.

POCHET, S.; DUGUE, L.; LABESSE, G.; DELEPIERRE, M.; MUNIER-LEHMANN, $\mathrm{H}$. Comparative study of purine and pyrimidine nucleoside analogues acting on the thymidylate kinases of Mycobacterium tuberculosis and of humans. ChemBioChem, v.4, p.742-747, 2003.

SOUZA, M. V. N.; VASCONCELOS, T. R. A. Fármacos no Combate à Tuberculose: Passado, Presente e Futuro. Química Nova, v. 28, n. 4, p.678-682, 2005.

VAN DAELE, I.; MUNIER-LEHMANN, H.; HENDRICKX, G. M.; CHAVAROT, P.; FROEYEN, M.; QING, L.; MARTINS, J.; VAN CALENBERGH, S. Synthesis and Biological Evaluation of Bicyclic Nucleosides as Inhibitors of Mycobacterium tuberculosis Thymidilate Kinase, ChemMedChem, v.1, p.1081-1090, 2006.

VAN DAELE, I.; MUNIER-LEHMANN, H.; FROEYEN, M.; BALZARINI, J.; VAN CALENBERGH, S. Rational design of 5'thiourea-substituted $\square$-thymidine analogues as thymidine monophosphate kinase inhibitors capable of inhibiting mycobacterial growth. Journal of Medicinal Chemistry, v.50, p.5281-5292, 2007.

VANHEUSDEN, V.; MUNIER-LEHMANN, H.; POCHET, S.; HERDEWIJN, P.; CALENBERGH, S. V. Synthesis and evaluation of thymidine-5'-O-monophosphate analogues as inhibitors of Mycobacterium tuberculosis thymidylate kinase. Bioorganic $\&$ Medicinal Chemistry Letters, v.12, p.26952698, 2002.

VANHEUSDEN, V.; MUNIER-LEHMANN, H.; FROEYEN, M.; DUGUE', L. HEYERICK, A.; KEUKELEIRE, D. D.; POCHET, S.; BUSSON, R.; HERDEWIJN, P.; CALENBERGH, S. V. 3'-C-branchedchain-substituted nucleosides and nucleotides as potent inhibitors of Mycobacterium tuberculosis thymidine monophosphate kinase. Journal of Medicinal Chemistry, v.46, p.3811-3821, 2003a.

VANHEUSDEN, V.; ROMPAEY, P. V.; MUNIER-LEHMANN, H.; POCHET, S.; HERDEWIJN, P.; CALENBERGH, S. V. Thymidine and Thymidine-5' -Omonophosphate analogues as Inhibitors of Mycobacterium tuberculosis Thymidylate Kinase. Bioorganic \& Medicinal Chemistry Letters, v.13, p.3045-3048, 2003 b.

WORLD HEALTH ORGANIZATION. Tuberculosis: Global Tuberculosis Control, 2010.[http://whqlibdoc.who.int/publications/2 010/9789241564069_eng.pdf 